\title{
Ein in der Onkologie noch stets unterschätztes Problem? Ärztemangel in Deutschland
}

\author{
Lik \\ Herimen \\ und terr,
}

der zukünftige Ärztemangel ist von der Politik als ernstzunehmendes Problem erkannt worden. Die nicht zuletzt auf dem Deutschen Ärztetag diskutierten Daten zeigen, dass es sich dabei um eine große Herausforderung handelt, deren Bewältigung nicht auf die lange Bank geschoben werden kann. Trotzdem wird die Problematik für den Bereich der Onkologie meines Erachtens immer noch unterschätzt.

\section{„Für das Jahr 2020 wird eine zunehmende Diskrepanz zwischen ärztlichem Angebot und medizinischer Nachfrage prognostiziert"}

Die zukünftige Versorgungssituation für die Onkologie ergibt sich aus der Zahl der in Zukunft zur Verfügung stehenden Onkologinnen und Onkologen (genauer aus der durch diese zur Verfügung gestellte Arbeitszeit) und dem Bedarf an Onkologen, der sich wiederum aus der Bevölkerungsentwicklung und aus der altersabhängigen Inzidenz und Prävalenz der onkologischen Erkrankungen ergibt.
Für die USA hat eine Task Force der ASCO für 2020 eine zunehmende Diskrepanz zwischen ärztlichem Angebot und medizinischer Nachfrage berechnet. Entsprechende Daten für Deutschland fehlen. Solch eine Analyse wäre für Deutschland aus meiner Sicht dringend notwendig.

Viel Freude beim Lesen dieser Ausgabe von best practice onkologie, Ihr

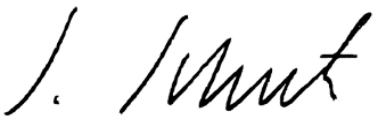

PD Dr. Stephan Schmitz Schriftleiter von best practice onkologie

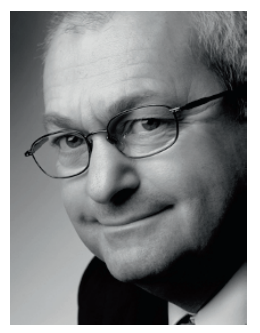

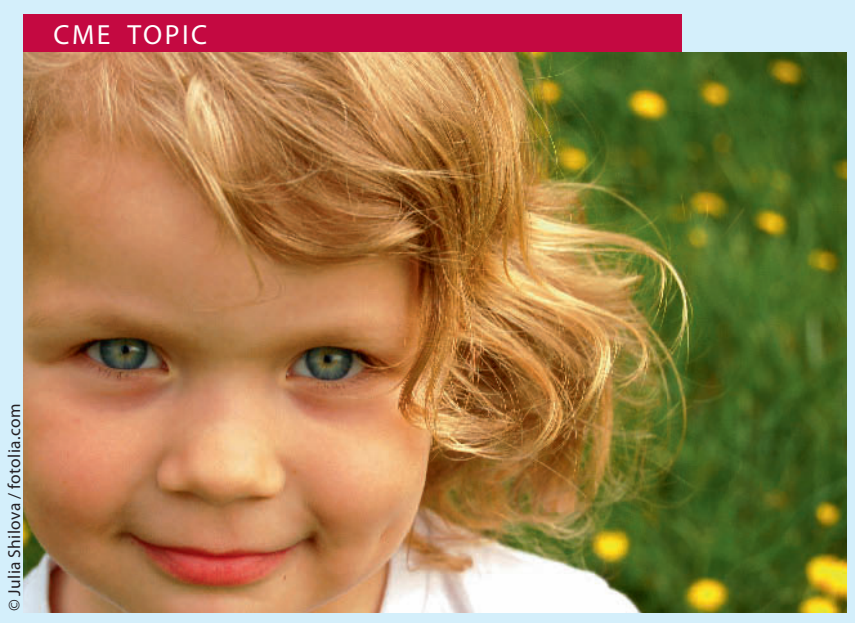

Schmerztherapie in der pädiatrischen Hämatologie und Onkologie

Schmerzen bei Kindern und Jugendlichen werden weltweit zu wenig beachtet. Dabei sind sie ein Risikofaktor für vielfältige gesundheitliche Probleme im Erwachsenenalter. Erfahren Sie mehr über Schmerzarten, Schmerzmessung und Schmerztherapie bei Kindern und Jugendlichen mit hämatologisch-onkologischen Erkrankungen ab Seite 44.

Nutzen Sie diesen Fortbildungsbeitrag, um drei Punkte zu sammeln!

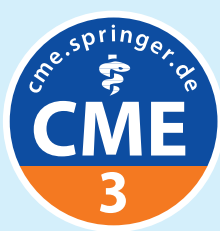

